\title{
Haystack Principle - Its Importance in Unearthing the Electro- cardiogram in a Patient with Unexplained Syncope
}

\author{
Santosh Kumar Sinha, FACC, FAESC, FSCAI* (D), Awadesh Kumar Sharma (D), Mahmodullah Razi \\ and Umeshwar Pandey
}

Department of Cardiology, LPS Institute of Cardiology, GSVM Medical College, India

*Corresponding author: Santosh Kumar Sinha, FACC, FAESC, FSCAI, Associate Professor, Department of Cardiology, LPS Institute of Cardiology, GSVM Medical College, Kanpur, Uttar Pradesh, 208002, India,

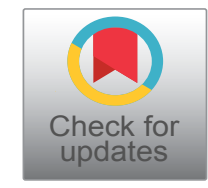
Tel: +91-9670220088, Fax: +91-0512-2556199; 2556521

\begin{abstract}
The "haystack principle" is of great diagnostic and therapeutic importance to unearth the obscure $P$ waves which gives clue to underlying diagnosis. Here, we report a case of 72-year-old women who came to outdoor department for evaluation of repeated episodes of loss of consciousness. Electrocardiogram revealed complete right bundle branch block pattern with bizarre T wave in lead II, III, and aVF. $P$ wave were not discernible. However, lead aVR revealed well formed $P$ wave with second-degree atrioventricular block. Patient was successfully managed with implantation of permanent pacemaker. Therefore, whenever one is faced with the problem of finding elusive $P$ wave, lead showing the smallest electrical deflection should always be carefully examined to clinch the diagnosis.
\end{abstract}

\section{Keywords}

Haystack principle, Atrioventricular block, Syncope, Right bundle branch block

\section{Introduction}

Syncope of unexplained reason can be mostly diagnosed using surface electrocardiogram (ECG) or else his bundle electrocardiogram (HBE). One should be careful in delineating $P$ and QRS. In evaluation of arrhythmia, QRS complex should be milked first as it gives maximum information and state of ventricular behaviour. If its behaviour is stable, atrial rhythm can be ignored. If it is bizarre, it gives information about its origin which may either supraventricular or ventricular. $\mathrm{P}$ preoccupation syndrome or "cherchez le $P$ " certainly plays a role. While identifying $P$ wave, there are several caveats and clue which be kept in mind.

\section{Case Report}

A 70-year-old, hypertensive male presented to outdoor patient department for evaluation of dizziness and syncope. His blood pressure before initiation of treatment was $160 / 90 \mathrm{mmHg}$ in left arm in supine position. He was receiving amlodipine $10 \mathrm{mg}$ and hydrochlorthiazide $12.5 \mathrm{mg}$. His blood pressure was $138 / 78 \mathrm{mmHg}$. Postural hypotension was ruled out. Other physical examinations were unremarkable. ECG revealed RBBB pattern with rate of 77 per minute. The top tracing rhythm strips (lead II, V1, and V4) showed RBBB pattern but QRS was bizarre (red circle; Figure 1). P was inverted and giving an impression of first degree heart block. However, T wave morphology was abnormal and wide. As his symptoms were classical of cardiac syncope, ECG demanded a careful look. Lead aVR provided a conclusive evidence of 2:1 atrioventricular (AV) conduction block which was of Mobitz type II. Every alternate $P$ wave was nonconductive (red arrow; Figure 1). Blocked $P$ waves were concealed in QRS complexes in lead I and in T waves in II, III, and V2-V6. It was best discernible in lead aVR only as ventricular complexes were smallest in this lead. This was the reason which made the $P$ wave best visible in lead aVR which followed "the haystack principle". His echocardiogram showed normal systolic function (ejection fraction-55\%) and grade-I diastolic dysfunction. His biochemistry including electrolytes, and other haematological parameters were unremar-

Citation: Sinha SK, Sharma AK, Razi M, Pandey U (2020) Haystack Principle - Its Importance in Unearthing the Electrocardiogram in a Patient with Unexplained Syncope. Int J Clin Cardiol 7:208. doi. org/10.23937/2378-2951/1410208

Accepted: December 10, 2020: Published: December 12, 2020

Copyright: (C) 2020 Sinha SK, et al. This is an open-access article distributed under the terms of the Creative Commons Attribution License, which permits unrestricted use, distribution, and reproduction in any medium, provided the original author and source are credited. 


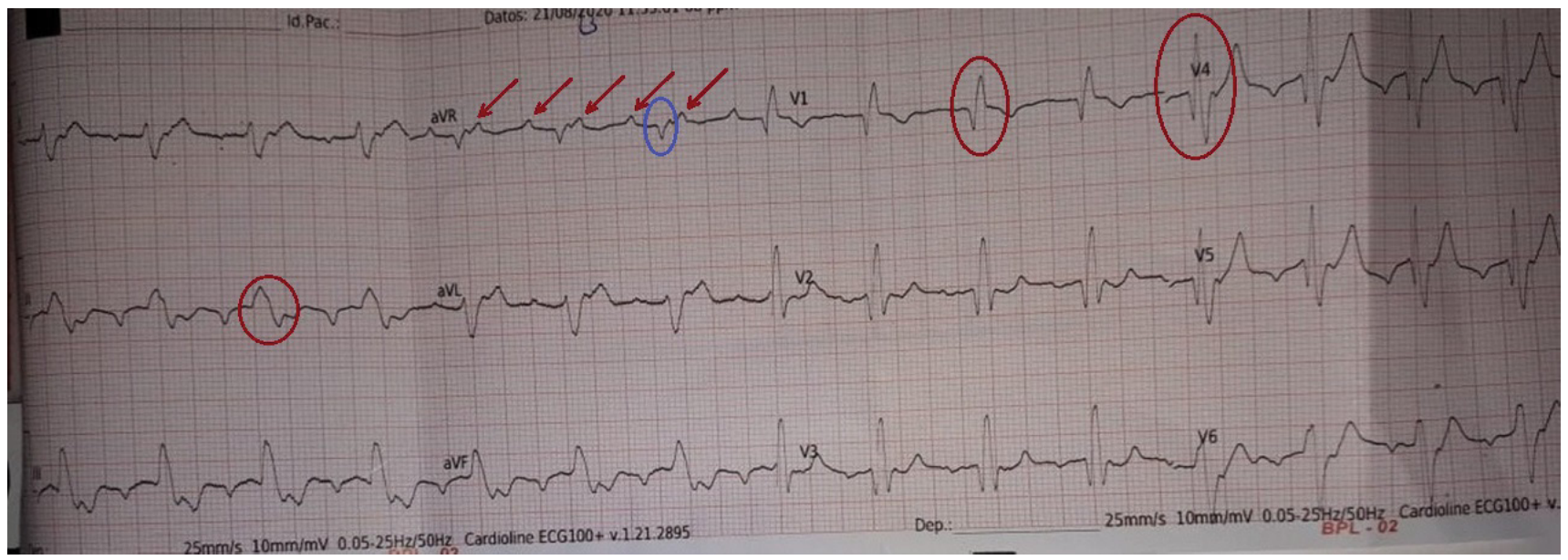

Figure 1: The rhythm strips (lead II, V1, and V4) showed RBBB pattern with bizarre QRS (red circle). Lead aVR provided a conclusive evidence of 2:1 atrioventricular (AV) conduction block which was of Mobitz type II. Every alternate $P$ wave was nonconductive (red arrow). Blue circle represents QRS complex in lead aVR.

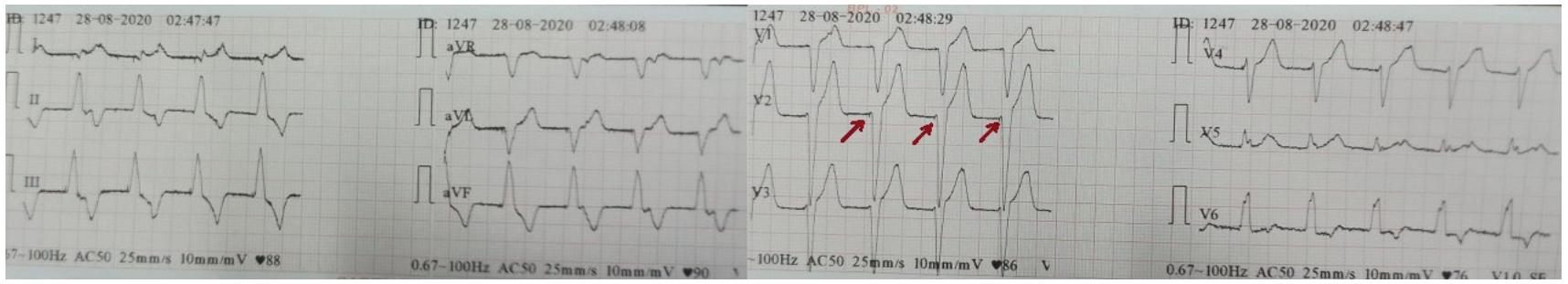

Figure 2: Pacing electrocardiogram showing left bundle branch morphology. Red arrow represents pacing spike.

kable. A permanent pacemaker (VVIR- StJude Medical; USA) was implanted from left side aseptically after obtaining written informed consent. It was sensing and pacing properly and all his electrical parameters were normal (Figure 2). He was discharged in stable condition with appropriate follow up advice.

\section{Discussion}

Most of the disturbances of rhythm and conduction are recognised by looking at QRS complexes alone. Supraventricular arrhythmias are characterized by normal QRS complexes except in cases of aberrancy. Ventricular origin of arrhythmia is always characterized by bizarre QRS complex with prolonged QRS complex. If the diagnosis is not apparent at first look, a systematic approach is always warranted. It starts with milking of QRS complex as its normal duration in at least two lead of ECG nearly rules out ventricular origin of arrhythmia. If it is abnormal, one is left with differentiating either ventricular origin or supraventricular tachyarrhythmia (SVT) with aberrancy [1]. Morphology of QRS complex in this situation plays a major role. If above is not conclusive, p wave should be analysed what is called as "Cherchez le $P$ " by Marriot. In case of obscure $P$ wave, it should be magnified by either placing the leads at different position than conventional one or one should look at the lead where it is best visible. Conventionally, rhythm strip (lead II, V1) gives maximum information but at times, these may not be informative like in our case. So- metimes, carotid sinus massage does a great help [2]. Another approach is known as "Bix rule" which states that whenever the P waves of SVT are halfway between QRS complexes, additional $P$ wave will be hiding within QRS complex. It may be better visualised using any vagal manuover but carries a potential of acceleration of ventricular rate. If $P$ wave is doubtful in cases of wide QRS complex in the background of bradyarrythmia, "Haystack Principal" offers a great help which states in order to find a needle in a haystack, smallest haystack should be searched first. In cases of an interloping ECG wave (extra P waves); a lead with the flattest deflection should be focussed [3]. Some leads (aVR) are intuitively appearing unhelpful while identifying sources of arrhythmia. In our case, had aVR would not been analysed, it would have been difficult to clinch the diagnosis. Lead aVR on the basis of haystack principle help to save the life of patient by pacemaker implantation.

\section{Conflict of Interest}

None.

\section{Sources of Support (If Applicable)}

Disclosure of funding received for this work from any of the following organizations: National Institutes of Health (NIH); Welcome Trust; and other(s) - None.

\section{Statement of Equal Authors' Contribution}

All authors made equal contribution. 


\section{References}

1. Spodick HD (2006) Second-degree atrioventricular block and the haystack principle. Am J Geriatr Cardiol 15: 66.
2. Meek S, Morris F (2002) Introduction II-Basic terminology: $A B C$ of clinical electrocardiography. BMJ 324: 470-473.

3. Hurst JW, Fye WB (2007) Henry J. L. Marriott: Lucid teacher of electrocardiography. Clin Cardiol 30: 207-208. 